\title{
Uso de redes neurais artificiais para predição de índices zootécnicos nas fases de gestação e maternidade na suinocultura
}

\author{
Héliton Pandorfi ${ }^{1}$, Iran José Oliveira Silva ${ }^{2}$, Valéria Cristina Rodrigues Sarnighausen ${ }^{3}$, \\ Frederico Márcio Corrêa Vieira ${ }^{3}$, Sheila Tavares Nascimento ${ }^{3}$, Cristiane Guiselini ${ }^{1}$
}

\footnotetext{
1 Departamento de Tecnologia Rural, UFRPE - NUPEA, R. Dom Manoel de Medeiros, s/n, 52171-900, Recife, PE, Brasil.

2 Departamento de Engenharia Rural, ESALQ/USP - NUPEA.

${ }^{3}$ Doutorando PPG em Física do Ambiente Agrícola, ESALQ/USP - NUPEA.
}

RESUMO - Objetivou-se com este trabalho avaliar a precisão das redes neurais artificiais (RNA) na estimativa das redes neurais artificiais (RNA) na predição de índices zootécnicos, com base em variáveis térmicas e fisiológicas de porcas gestantes. A pesquisa foi realizada entre janeiro e abril de 2005 em uma propriedade de produção industrial de suínos, no setor de gestação, com 27 matrizes primíparas, alojadas em baias individuais e posteriormente na maternidade em baias de parição, onde foram quantificados os índices de produção dos leitões provenientes do estudo. Para tanto, foi implementada uma RNA backpropagation, com uma camada de entrada, uma oculta e uma camada de saída com funções de transferência tangente sigmoidal. A temperatura do ar e a frequência respiratória foram consideradas variáveis de entrada e o peso ao nascimento dos leitões e número de leitões mumificados, como variáveis de saída. A rede treinada apresentou ótimo poder de generalização, o que possibilitou a predição das variáveis-respostas. A caracterização do ambiente da gestação e maternidade foi adequada se comparada aos dados reais, com poucas tendências de sub ou superestimação de alguns valores. A utilização desse sistema especialista para a previsão dos índices zootécnicos é viável, pois o sistema tem bom desempenho para esta aplicação.

Palavras-chave: sistemas especialistas; suinocultura; zootecnia de precisão

\section{Use of artificial neural networks on the prediction of zootechnical indexes on gestation and farrowing stages of swines}

\begin{abstract}
The objective of this work was to evaluate the precision of Artificial Neural Networks (ANNs) to estimate zootechnical indexes, based on thermal and physiological variables of pregnant sows. This study was carried out from January to April 2005, in a swine industrial production farm in the gestation section with 27 primiparous gilts, allocated in individual pens and after on farrowing pens where it was quantified animal production indexes of piglets from the study. Therefore, an ANN backpropagation was implemented, with one input layer, one hidden layer, and one output layer with tangent sigmoidal transference functions. Air temperature and respiratory frequency were considered as input variables and weight of piglet at birth and the number of mummified piglets as output variables. The trained ANN presented a great generalization power, which enabled the prediction of the answer-variables. Characterization of the environment of gestation and maternity was appropriated if compared to the real data, with few under or overestimated tendencies of some values. The use of this specialist system to predict zootechnical indexes is viable because the system shows a good performance for this use.
\end{abstract}

Key Words: animal production precision, specialist systems, swine production

\section{Introdução}

Com o avanço da tecnologia da informação, a utilização de sistemas especialistas para predição de parâmetros produtivos torna-se cada vez mais importante no gerenciamento agropecuário e destaca-se como ferramenta na tomada de decisão. A aplicabilidade das redes neurais artificiais é crescente e a redução no custo de implantação é constante à medida que se popularizam ferramentas computacionais que permitem a inserção de novas técnicas para solução de problemas complexos, como a predição de dados não-lineares e padrões de comportamento. A entrada e saída de dados dos sistemas produtivos são preditas nas análises múltiplas e paralelas das redes (Murase, 2000).

As redes neurais artificiais são modelos de processamento serial ou distribuídos paralelamente, procurando alcançar bom desempenho via interconexão de elementos computacionais simples. Apesar de desenvolver 
um programa de instruções sequenciais, os modelos exploram simultaneamente muitas hipóteses utilizando regras de aprendizagem que permitem adquirir poder de generalização suficiente para reconhecer padrões e predizer cenários (Loesch \& Sari, 1996; Haykin, 2001). Os modelos de redes neurais têm potencial de aplicação na produção animal quando é necessário um banco de dados que permita a compreensão das relações entre o ambiente e a exploração agropecuária (Shao et al., 1998; Pandorfi et al., 2006; Fernandez et al., 2006; Rodrigues et al., 2007; Vieira et al., 2010).

As principais vantagens de utilização dessas redes são a tolerância a falhas, a aplicação em tempo real, a capacidade de autoadaptação e a rápida resolução de problemas práticos, sem necessidade de definição de listas de regras ou de modelos precisos. Logo, o objetivo de definição da rede é resolver o problema com a menor estrutura possível, com potencial de aplicação em situação que requeiram classificação de padrões, identificação e associação de padrões, aproximação de funções e aprendizado em áreas em que é difícil criar modelos precisos da realidade e com frequentes mudanças de ambiente (Haykin, 2001).

Entre os potenciais de aplicação das redes neurais na agropecuária, destacam-se o controle dos elementos meteorológicos em instalações zootécnicas como resposta ao comportamento postural, à vocalização e o consumo de energia metabolizável; a adequação do manejo com base nas variáveis fisiológicas, nos aspectos nutricionais e na resposta produtiva; a caracterização da sanidade do rebanho sem a utilização de práticas invasivas; e a seleção e melhoramento genético e o monitoramento de operações de pré-abate na determinação da qualidade do produto final.

Dessa forma, objetivou-se com este trabalho avaliar o desempenho das redes neurais artificiais (RNA) na predição de índices zootécnicos com base em variáveis térmicas e fisiológicas de porcas gestantes.

\section{Material e Métodos}

A pesquisa foi realizada em uma granja comercial, localizada no município de Elias Fausto, Estado de São Paulo, a uma latitude de $22^{\circ} 36^{\prime} \mathrm{S}$, longitude de $47^{\circ} 36^{\prime} \mathrm{W}$ e altitude de $535 \mathrm{~m}$. O clima da região é caracterizado como mesotérmico Cwa (tropical de altitude), com temperatura do mês mais frio entre 3 e $18^{\circ} \mathrm{C}$, inverno seco e temperatura do mês mais quente maior que $22^{\circ} \mathrm{C}$, segundo classificação Köppen (Pereira et al., 2002).

A instalação referente ao confinamento das matrizes gestantes apresentava dimensões de $15 \times 60,0 \mathrm{~m}$, pé-direito de $2,3 \mathrm{~m}$, beiral de $0,5 \mathrm{~m}$, orientação leste-oeste e cobertura com telhas cerâmicas do tipo francesas. Foram confinadas na estrutura 380 matrizes alojadas em baias individuais com dimensões de 1,95 $\mathrm{m}$ de comprimento por 0,60 $\mathrm{m}$ de largura e 1,20 m de altura, totalizando uma área de $1,17 \mathrm{~m}^{2}$. Desses animais, foram amostrados aleatoriamente 27 para composição do banco de dados.

O estudo foi realizado com animais Landrace $\times$ Large White, de mesma ordem de parto (primíparas), com o objetivo de eliminar possíveis fontes de variação no estudo, como habilidade materna, por exemplo. Todos os índices zootécnicos (peso dos leitões ao nascimento e número de leitões mumificados) determinados nos animais controle foram fornecidos pela granja.

As variáveis de entrada utilizadas na construção da rede foram temperatura do ar $\left({ }^{\circ} \mathrm{C}\right)$ e taxa respiratória (movimentos/minuto), determinadas durante a gestação, e as de saída foram peso ao nascimento dos leitões e número de leitões mumificados, determinados na maternidade. A temperatura do ar e frequência respiratória foram registradas semanalmente, às 9 h, 12 h e 17 h, no período entre 4/1/2005 e 27/4/2005, ou seja, durante 114 dias.

Foi gerado e caracterizado um banco de dados, que foi usado no desenvolvimento, treinamento e na validação da RNA para estimativa dos índices zootécnicos. Todos os dados foram normalizados $[0,1]$ com o objetivo de facilitar a convergência durante o processo de treinamento da rede, por meio da seguinte equação:

$\mathrm{y}=\frac{\left(\mathrm{y}_{0}-\mathrm{y}_{\min }\right)}{\left(\mathrm{y}_{\max }-\mathrm{y}_{\min }\right)}$

em que: $\mathrm{y}_{0}$ é o valor a normalizar; $\mathrm{y}_{\max }$ e $\mathrm{y}_{\min }$ são valores mínimos e máximos entre os valores reais das variáveis.

$\mathrm{O}$ algoritmo de aprendizado utilizado neste trabalho para desenvolvimento da rede foi o backpropagation (BP), citado por Haykin (2001), a partir da generalização da regra de aprendizado Widrow-Hoff, para redes do tipo feedfoward perceptron. A regra de aprendizado WidrowHoff, também conhecida como Regra Delta - LMS (minimização do erro médio quadrático) ajusta os pesos das conexões entre os neurônios da rede de acordo com o erro e tem por objetivo encontrar um conjunto de pesos e polarizações que reduzam a função erro.

A topologia da arquitetura da rede backpropagation foi formada por uma camada de entrada, uma escondida (oculta) de neurônios não-lineares e outra de saída de neurônios com função de transferência tangente sigmoidal (Figura 1).

Essas camadas foram utilizadas na aproximação das variáveis temperatura do ar e frequência respiratória e dos índices zootécnicos (peso no nascimento dos leitões e 


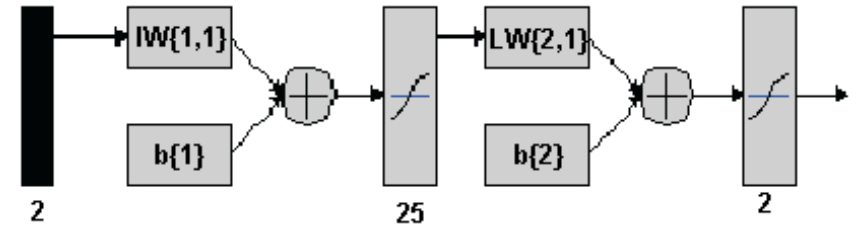

Figura 1 - Arquitetura da rede empregada no treinamento.

número de leitões mumificados), cuja matriz de entrada foi de $27 \times 2$ (27 observações com 2 variáveis) e a de saída, 27 $\times 2$. Segundo um critério heurístico, escolheu-se a seguinte topologia: 25 neurônios para a camada escondida e 2 para a camada de entrada e saída (Tabela 1).

O algoritmo backpropagation para o treinamento da rede foi descrito pelos seguintes passos:

- passo 1 - iniciar os pesos, polarizações e demais parâmetros de treinamento;

- passo 2 - apresentar à rede um padrão de entrada do conjunto de treinamento composto de entradas e saídas;

- passo 3 - calcular o erro para os neurônios da camada de saída $\left(e_{k}\right)$, subtraindo a saída desejada da saída calculada (processamento interno da rede);

$e_{k}=\left(y_{k}-\hat{y}_{k}\right)$

em que: $y$ = saída desejada; $\mathrm{e} \hat{y}$ = saída real (saída gerada pela rede).

- passo 4 - calcular o ajuste nos pesos da camada da saída $\left(\Delta w_{i}^{0}\right)$ por meio do seguinte modelo:

$\Delta w_{i}^{o}(k+1)=-\frac{\partial e_{k}^{2}}{\partial w_{i}^{o}(k+1)}=\eta \cdot\left(y_{k}-\hat{y}_{k}\right) \cdot p^{\prime}\left(n e t^{0}(k)\right)$.

.$\left(w_{i}^{o}(k), O_{i}^{1}(k)\right)$

em que: $p$ = função contínua derivável, tangente hiperbólica; $\eta$ = taxa de aprendizagem; net = estado de ativação (funções de ativação); e $O_{i}^{1}=$ entrada.

- passo 5 - retropropagar o erro para as camadas escondidas. Como não existe uma saída desejada para os neurônios das camadas escondidas, calculou-se o erro a partir do erro dos neurônios pertencentes à camada de saída e das conexões que os interligam. Obteve-se a seguinte equação para calcular o ajuste dos pesos para a primeira camada escondida mais próxima à saída.

Tabela 1 - Parâmetros de treinamento utilizados para o algoritmo backpropagation

\begin{tabular}{lc}
\hline Parâmetro & Valor \\
\hline Taxa de aprendizagem & 0,1 \\
Erro & 0,005 \\
Neurônio na camada oculta & 25 \\
Função de transferência na camada oculta & Tangente sigmoidal \\
Função de transferência na camada de saída & Tangente sigmoidal \\
\hline
\end{tabular}

$$
\begin{aligned}
& \Delta w_{j, i}^{1}(k+1)=-\frac{\partial e_{k}^{2}}{\partial w_{j, i}^{1}(k+1)}=\eta \cdot\left(y_{k}-\hat{y}_{k}\right) p^{\prime}\left(n e t^{o}\right) . \\
& \frac{\partial a}{\partial O_{j}^{1}(k)}\left(w_{j}^{o}(k), O_{j}^{1}(k)\right) \cdot p^{\prime}\left(n e t_{j}^{1}\right) \cdot \frac{\partial a}{\partial w_{j, i}^{1}(k)} \\
& \left(w_{j, i}^{1}(k), x_{i}(k)\right)
\end{aligned}
$$

- passo 6 - calcular o erro acumulado da rede. Nesta etapa, verificou-se se o erro total sobre todos os padrões de entrada pode ser considerado desprezível, isto é, abaixo de um limiar de aceitação. Nesse caso, o algoritmo deve parar, caso contrário, volta-se ao passo 2.

$\mathrm{Na}$ avaliação do desempenho da rede para o teste e a validação da RNA, utilizou-se o conjunto de dados registrados na propriedade, sendo que $70 \%$ dos dados foram utilizados para o treinamento e $30 \%$ para sua validação (Prechelt, 1994; Haykin, 2001). O critério adotado para parada do treinamento foi uma combinação de métodos, pelo erro e pelo número de ciclos (epochs), encerrando-se o treinamento quando um dos critérios foi atendido. $\mathrm{Ou}$ seja, o treinamento foi encerrado quando houve convergência na superfície do erro médio quadrático $(0,005)$, que consistiu numa análise de performance de predição da rede. Por meio desse erro, esperaram-se valores próximos de zero, visando ao desempenho satisfatório da rede.

Para resolução das etapas subsequentes, utilizou-se o Neural Network Toolbox do Matlab ${ }^{\circledR}$ 6.5, aplicando-se a ferramenta computacional exclusiva para redes neurais artificiais, que possui um conjunto de funções predefinidas.

\section{Resultados e Discussão}

Para efeito de treinamento da rede, adotou-se o parâmetro de taxa de aprendizado de 0,1 , uma vez que valores superiores a este não permitiram convergência do processo na superfície do erro médio quadrático. O parâmetro taxa de aprendizado teve grande influência durante o processo de treinamento da rede neural. Taxas menores tornaram o aprendizado da rede muito lento, ao passo que uma taxa de aprendizado muito alta provocou oscilações no treinamento e impediu a convergência do processo de aprendizado, tornando-se recomendáveis valores de 0,1 a 1,0.

Um dos problemas no treinamento da rede neural tipo Multi Layer Perceptron (MLP) com treinamento backpropagation é a definição de seus parâmetros. A seleção dos parâmetros de treinamento do algoritmo é um processo que demandou grande esforço, pois pequenas diferenças nesses parâmetros levaram a grandes alterações, tanto no tempo de treinamento como na generalização 
obtida. Xin (1999) afirmou que os melhores modelos são aqueles que possuem medidas ou parâmetros mais objetivos. Nesse caso, o uso de técnicas estatísticas pode facilitar a escolha destas variáveis de entrada, selecionando aquelas cujos pesos são determinantes no alcance do poder de generalização desejável das redes (Sanzogni \& Kerr, 2001; Kominakis et al., 2002; Vieira et al., 2010). Além disso, Lacroix et al. (1997) encontraram melhorias no desempenho das redes quando as variáveis recebiam algum tratamento prévio, principalmente em categorias com pequeno número de dados. Afirmaram ainda que a arquitetura backpropagation apresentou maior sensibilidade ao preparo dos dados, quando comparada às demais topologias . Assim, a escolha de variáveis antes do treino da rede possibilita que informações representativas contribuam com o aprendizado, aumentando a acurácia no reconhecimento de padrões.

O maior do número de camadas elevou a complexidade e o tempo de processamento da rede. A quantidade elevada de neurônios por camada resultou em aumento do grau de liberdade da função de transferência e, quanto maior a quantidade de variáveis livres, menor o poder de generalização. Com isso, após inúmeros ajustes em função do resultado, foi alcançado poder de generalização adequado com 524 ciclos (épocas), ou seja, o número de vezes em que o conjunto de treinamento foi apresentado à rede.

$\mathrm{O}$ conjunto de treinamento foi apresentado à rede neural para que o algoritmo backpropagation retropopagasse os erros (Figura 2). Isso significa que o

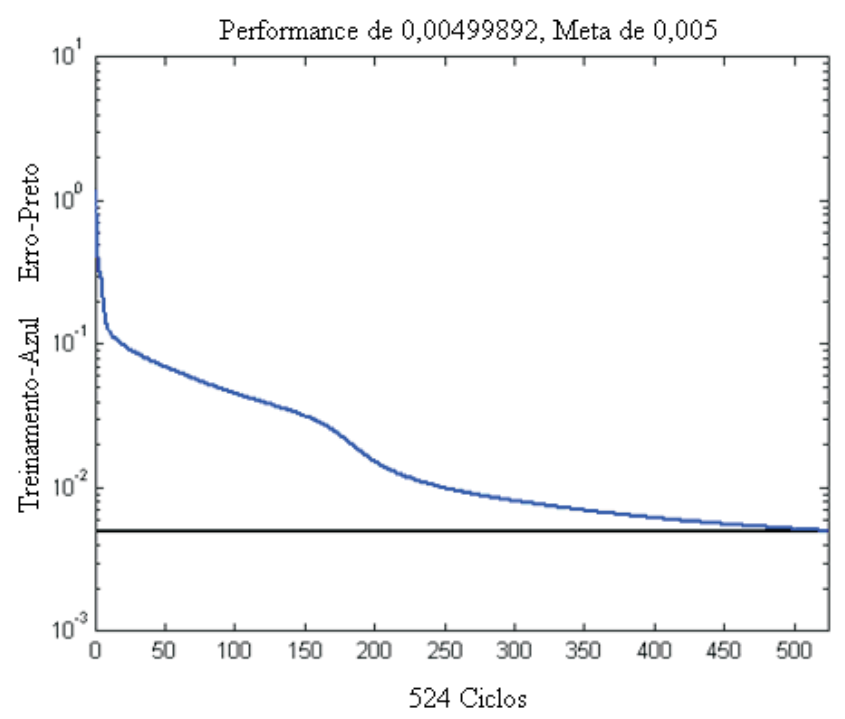

Figura 2 - Curva de treinamento da rede para atualização dos pesos sinápticos para estimativa dos índices zootécnicos. algoritmo realizou várias interações para a atualização dos pesos sinápticos até atingir o valor de erro $(0,005)$.

O treinamento foi satisfatório e a rede convergiu para o valor do erro determinado (Tabela 1) apresentando precisão da ordem de 0,005 (valor exato de 0,00499 ). As previsões giram em torno de um valor médio capaz de simular os picos dos eventos (peso ao nascimento e número de leitões mumificados). O modelo de previsão, portanto, explicou a variação do peso no nascimento dos leitões, tendo em vista o valor encontrado para o coeficiente de determinação $\mathrm{R}^{2}=0,9389$ (Figura 3a). Esse fato reforça a possibilidade de utilização das variáveis de entrada (temperatura do ar e frequência respiratória) para predição do peso ao nascimento dos leitões, caracterizando-se como indicativo de alerta à condição de estresse térmico e um parâmetro suporte na tomada de decisão.

O sucesso obtido pela rede corrobora estudos realizados por Kominakis et al. (2002) para predição da produção diária de leite em ovelhas em lactação com uso de RNA, nos quais foi obtido adequado planejamento do fluxo de produção com base nos parâmetros fisiológicos e ambientais. Xin (1999) desenvolveu um sistema automatizado de análise de imagens, que faz os ajustes térmicos apropriados para melhorar o bem-estar de leitões e a sua eficiência produtiva, usando o comportamento postural como entrada em uma rede neural de retropropagação com três camadas, que foi treinada para classificar o estado de conforto térmico.

Em geral, as aproximações dos valores estimados pela rede caracterizaram adequadamente os dados de registro real, evidenciando somente alguns pontos isolados em que houve pequena tendência a subestimar valores altos ou superestimar valores baixos (Figura 3b). Salehi et al. (1998) afirmaram que essas variações são ocasionadas pela presença de valores discrepantes no conjunto de dados e influenciam a predição das variáveis respostas.

Segundo Kominakis et al. (2002), o desempenho da rede poderia ser melhorado com a utilização de um conjunto maior de dados na fase de treinamento, possibilitando direcionar os parâmetros específicos de treinamento e permitindo melhor qualidade da predição. A habilidade de se predizer variadas situações aumenta proporcionalmente ao conjunto de dados (Jaiswal et al., 2005). Isso pode ser explicado pelas informações úteis contidas em um conjunto de dados representativo que potencializam o reconhecimento de padrões. No entanto, Betker et al. (2003) afirmaram que a existência de lacunas nos dados de um fator pode causar incerteza no sistema, isto é, a predição apresenta erros na relação entre a variável resposta e o 


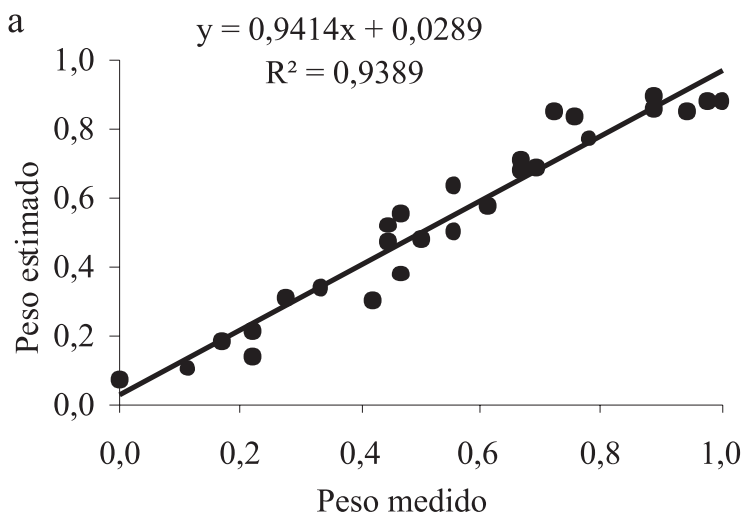

b

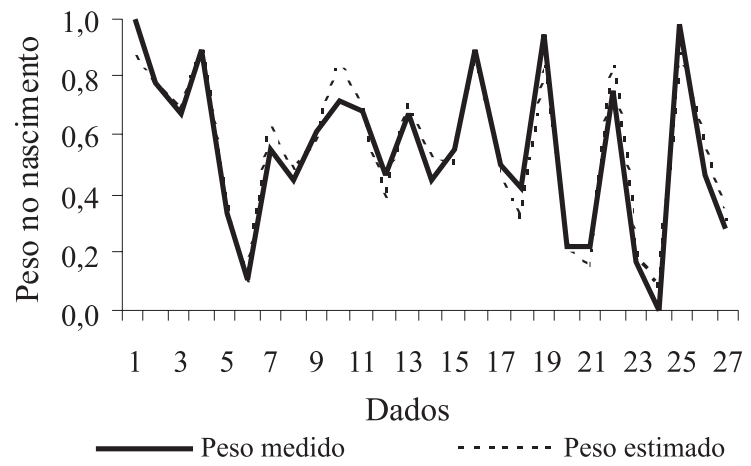

Figura 3 - Relação funcional para o peso dos leitões medidos e estimados pela rede (a) e sua variação na fase de teste e validação (b).

fator explicativo na falta de dados para o aprendizado. Os autores consideraram ainda que, nesses casos, a adição de mais informações na combinação de fatores pode melhorar a acurácia da rede. Ou seja, o aumento de variáveis de entrada, bem como a qualidade e a importância das mesmas para determinadas respostas a serem preditas, também pode melhorar o desempenho das redes (Lacroix et al., 1997; Park et al., 1998; Kominakis et al., 2002; Vieira et al., 2010).

Os pares de entradas e saídas possibilitaram evolução do aprendizado por meio da comparação entre a saída desejada e a real. As predições permitem simular o número de leitões mumificados com base nas variáveis de entrada, tendo em vista o valor encontrado para o coeficiente de determinação $\mathrm{R}^{2}=0,9526$ (Figura 4a).

A variação do número de leitões mumificados reais e estimados apresentou valores semelhantes, indicando generalização adequada dos dados medidos, uma vez que, em alguns pontos isolados, o mesmo perfil de variação foi observado para peso ao nascimento, denotando pequena tendência a subestimar valores altos ou superestimar valores baixos (Figura 4b).

Experiências anteriores também apontaram bom desempenho de redes neurais com o intuito de modelar matematicamente a resposta dos animais ao ambiente de criação. Roush et al. (1997) utilizaram a rede neural como predição probabilística (probabilistic neural network, PNN), que foi treinada para predizer ascite em frangos de corte, com base em fatores minimamente invasivos e relataram que o uso dos modelos desenvolvidos foi eficiente no diagnóstico e permitiu a escolha de linhagens que não tinham propensão a desenvolver ascite.

Roush \& Cravener (1997) compararam dois tipos de redes neurais artificiais, retropropagação e rede neural de regressão geral, para predição de níveis de aminoácidos em ingredientes alimentares e observaram que as redes neurais tiveram melhor desempenho em comparação à análise de
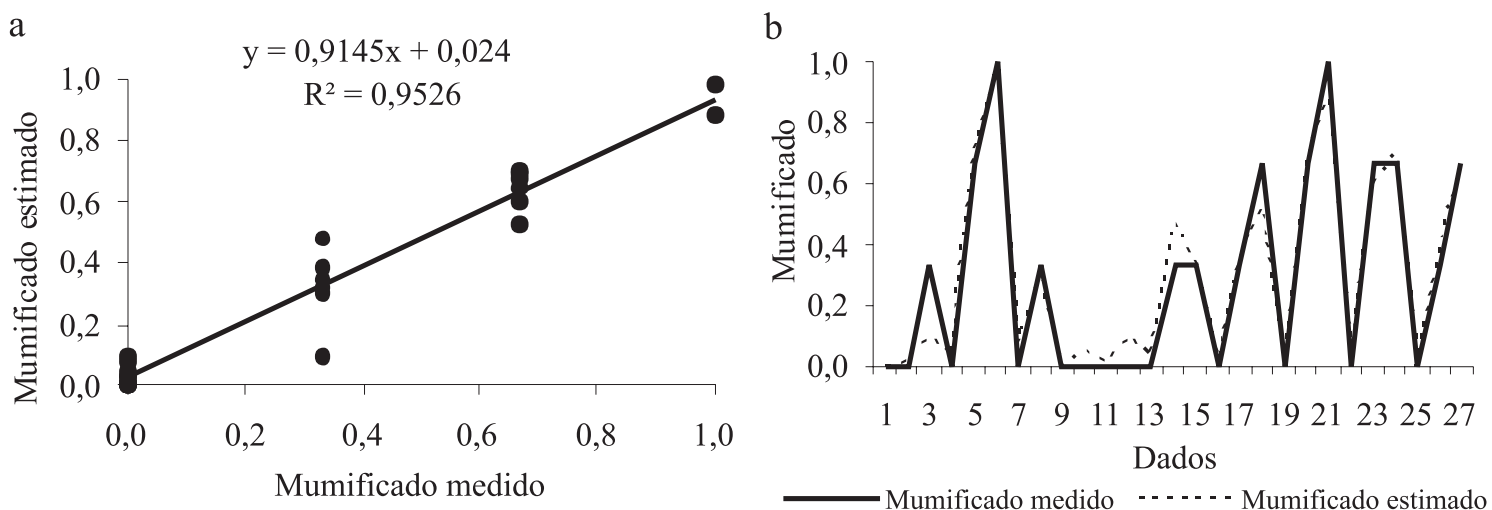

Figura 4 - Relação funcional para o número de leitões mumificados medidos e estimados pela rede (a) e sua variação na fase de teste e validação (b). 
regressão e que a rede neural de regressão geral superou a de retropropagação.

As variáveis ambientais e o manejo influenciam negativamente a eficiência reprodutiva. Assim, altas temperaturas, e demais fatores estressantes, tendem a ocasionar diminuição da fertilidade das fêmeas suínas, aumento na duração do parto, alta porcentagem de retorno ao cio, má-formação dos conceptos e maior mobilização de gordura corporal durante a lactação (Peltoniemi et al., 1999; Love et al., 1995), principalmente nas fêmeas primíparas (Vieira \& Vieira, 1987). Esses indicadores possibilitaram a obtenção de bom desempenho da rede para predição dos índices zootécnicos.

É importante ressaltar que outras arquiteturas de rede ou outros parâmetros também podem ser aplicados para situações semelhantes e que a solução proposta neste trabalho foi escolhida com o objetivo de apresentar o potencial de aplicação da ferramenta e seu bom desempenho nesse tipo de problema.

\section{Conclusões}

A utilização de redes neurais artificiais é viável para predição dos índices zootécnicos (peso ao nascimento dos leitões, número de leitões mumificados) com base nas variáveis temperatura do ar e frequência respiratória de fêmeas gestantes como representantes da condição de conforto térmico e bem-estar animal. A topologia adotada (Figura 1) permite classificação satisfatória, devido à grande capacidade de convergência do algoritmo de aprendizagem backpropagation.

\section{Referências}

BETKER, A.L.; SZTURM, T.; MOUSSAVI, Z. Application of feedforward backpropagation neural network to center of mass estimation for use in a clinical environment. In: ANNUAL INTERNATIONAL CONFERENCE OF THE IEEE EMBS, 25., 2003, Cancun. Proceedings... Cancun: IEEE EMBS, 2003. p.17-21.

FERNANDEZ, C. Neural networks for animal science applications: Two case studies. Expert Systems with Applications, v.31, n.2, p.444-450, 2006.

HAYKIN, S. Redes neurais: princípios e práticas. Tradução ENGEL, P.M. 2.ed. Porto Alegre: Bookman, 2001. 900p.

JAISWAL, S.; BENSON, E.R.; BERNARD, J.C. et al. Neural network modelling and sensitivity analysis of a mechanical poultry catching system. Biosystems Engineering, v.92, n.1, p.59-68, 2005.

KOMINAKIS, A.P.; ABAS, Z.; MALTARIS, I. et al. A preliminary study of the application of artificial neural networks to prediction of milk yield in dairy sheep. Computers and Eletronics in Agriculture, v.35, p.35-48, 2002.

LACROIX, R.; SALEHI, F.; YANG, X.Z. et al. Effects of data preprocessing on the performance of artificial neural networks for dairy yield prediction and cow culling classification. Transactions of the ASAE, v.40, n.3, p.839-846, 1997.

LOESCH, C.; SARI, S.T. Redes neurais artificiais: fundamentos e modelos. Blumenal: FURB, 1996. 166p.

LOVE, R.J.; KLUPIEC, C.; THORNTON, E.J. et al. An interaction between feeding rate and season affects fertility of sows. Animal Reproduction Science, v.39, p.275-284, 1995.

MURASE, H. Editorial: Artificial intelligence in agriculture. Computers and Electronics in Agriculture, v.29, p.1-2, 2000.

PANDORFI, H.; SILVA, I.J.O.; CARVALHO, J.L. et al. Estudo do comportamento bioclimático de matrizes suínas alojadas em baias individuais e coletivas, com ênfase no bem-estar animal na fase de gestação. Engenharia Rural, v.17, n.1, p.1-10, 2006.

PARK, B.; CHEN, Y.R.; NGUYEN, M. Multi-spectral image analysis using neural network algorithm for inspection of poultry carcasses. Journal of Agricultural Engineering Research, v.69, p.351-363, 1998.

PELTONIEMI, O.A.T.; LOVE, R.J.; HEINOMEN, M. et al. Seasonal and management effects on fertility of the sow: a descriptive study. Animal Reproduction Science, v.55, p.47-61, 1999.

PEREIRA, A.R.; ANGELOCCI, L.R.; SENTELHAS, P.C. Agrometeorologia: fundamentos e aplicações práticas. Guaíba: Agropecuária, 2002. 478p.

PRECHELT, L. PROBEN1 - A set neural network benchmark problems and benchmarking rules. Württemberg: Karlsruhe Institut für Technologie, 1994. 37p. (Technical Report, 21).

RODRIGUES, V.C.; BARBOSA FILHO, J.A.D.; SILVA, I.J.O. Visão computacional: distribuição espacial de aves poedeiras em condições de conforto e estresse. Engenharia Rural, v.18, p.9-16, 2007.

ROUSH, W.B.; CRAVENER, T.L. Artificial neural network prediction of amino acid levels in feed ingredients. Poultry Science, v.76, n.5, p.721-727, 1997.

ROUSH, W.B.; KIRBY, Y.K.; CRAVENER, T.L. et al. Artificial neural network prediction of ascite in broilers. Poultry Science, v.75, p.1479-1487, 1996.

SALEHI, F.; LACROIX, R.; WADE, K.M. Effects of learning parameters and data presentation on the performance of backpropagation networks for milk yield prediction. Transactions of the ASAE, v.41, n.1, p.253-259, 1998

SANZOGNI, L.; KERR, D. Milk production estimates using feed forward artificial neural networks. Computers and Electronics in Agriculture, v.32, p.21-30, 2001

SHAO, J.; XIN, H.; HARMON, J.D. Comparison of image feature extraction for classification of swine thermal comfort behavior. Computers and Electronics in Agriculture, v.19, p.223-232, 1998.

VIEIRA, H.P.; VIEIRA, R.P. Definition of the summer infertility problem in the pig. Luxemburg: Commission of the European Communities, 1987. 35p.

VIEIRA, F.M.C.; SILVA, I.J.O.; BARBOSA FILHO, J.A.D. et al, Productive losses on broiler preslaughter operations: effects of the distance from farms to abattoirs and of lairage time in a climatized holding area. Revista Brasileira de Zootecnia, v.39, p.2471-2476, 2010.

XIN, H. Assessing swine thermal comfort by image analysis of postural behaviors. Journal of Animal Science, v.77, suppl.2, p.1-9, 1999. 